\title{
Energy Efficient Event-Based Networking for the Internet of Things
}

\author{
Panayiotis Kolios, Georgios Ellinas, Christos Panayiotou, and Marios Polycarpou
}

\begin{abstract}
Autonomy is a key feature for sustainable operation of the Internet of Things (IoT), since the large deployment scale of IoT devices makes management a very challenging task. For that reason, energy efficiency has become a primary design consideration in emerging and future IoT solutions. Already, beaconing and discontinuous transmission/reception is included in the upcoming IoT standards to enable devices to duty cycle between the active and sleep states in order to save energy. In many cases however, this periodic mode of operation results in unnecessary wake-ups that greatly reduce energy efficiency.

Event-based communication greatly outperforms periodic triggering in efficiency, since wake-ups occur only when particular events take place. The networking aspects of this promising approach are investigated herein with devices exchanging events for monitoring and control purposes. Both centralized optimal and distributed heuristic strategies are formulated to intelligently adjust the number of wake-up events in order to maximize energy efficiency. Extensive simulations are conducted to analyze the performance of the proposed strategies.
\end{abstract}

Index Terms-Event-based communication, Networking, Energy Efficiency, Internet of Things

\section{INTRODUCTION}

The Internet of Things is set to transform every economic sector by enabling monitoring and control of physical objects, systems, and processes through a myriad of interconnected devices [1]. To date, there are as many IoT enabled connections as there are consumer electronics, while this number is set to increase dramatically over the coming years [2]. Evidently, managing all these devices is becoming a very challenging task, especially in those cases that devices are battery operated and mobile. Moreover, the operating costs may become non-negligible as penetration grows and resource utilization (including energy and bandwidth consumption) increases. Hence, operational efficiency is becoming a primary design consideration in order to improve autonomy and sustain future growth.

To attain higher energy efficiency gains in communication, upcoming standards introduce discontinuous transmission/reception cycles with short wake-ups and extended deep sleep periods. For instance, the IEEE 802.3az standard [3] for energy-efficient Ethernet, powers down the transmitters when no exchange of data is necessary and periodically probes the communication link to check if there is pending data traffic to exchange. Similarly, the upcoming IEEE 802.11ah

P. Kolios, G. Ellinas, C. Panayiotou and M. Polycarpou are members of the KIOS Research Center for Intelligent Systems and Networks and the Department of Electrical and Computer Engineering, University of Cyprus, e-mail:\{pkolios, gellinas, christosp, mpolycar\}acy - ac. cy

978-1-5090-4130-5/16/\$31.00 2016 IEEE low power WiFi standard [4] and the LTE-MTC [5] cellular amendment (both purposefully designed for the IoT) define extended power down periods to conserve energy, while quick wake-ups are used to beacon data and check for connection requests. Doing so eliminates standby times, as well as times of inactivity during data exchange, in order to improve resource utilization. Unfortunately, periodic triggering of wakeups becomes a major source of inefficiency in this case, since energy is consumed unnecessarily when there is no new information to communicate and when no connection requests are made.

Event-based communication is a promising approach to remedy this problem by allowing communication circuitry to wake up only when particular events take place. These could be discrete events (e.g., on/off switching, change in state, etc) [6] or abnormal behavior events detected using a nominal model. Noticeably, the latter category received considerable attention recently by the research community through the realm of Big Data, whereby data analytics are employed to extract behavior patterns that are in turn used to gain knowledge and enable intelligent decision making [7].

In line with these efforts, we consider hereafter the deployment of IoT devices to manage systems that exhibit recurrent patterns for which a behavioral model can be obtained. In practice this model can be used to estimate the system and avoid continuous or periodic communication required to learn the system state. Communication events are only necessary when some abnormal change in the system state has occurred for which the model cannot anticipate. With such a model at hand, a variety of techniques can be used (including threshold checking, change checking, etc) to design event detection policies that balance tracking accuracy with the number of events communicated.

This work moves a step further and considers the network energy efficiency when interconnecting devices employing event-based communication. As noted above, devices power up the communication circuitry to exchange data only when a particular event has been triggered, and every other time communication circuitry remains asleep to conserve energy. Noticeably, when all interconnected devices operate in this way, then each device that needs to communicate its data has to wait until the destination wakes up and becomes available before establishing a new connection. Waiting greatly impacts energy efficiency and thus the number of wake-up events has to be properly adjusted to achieve the maximum energy efficiency.

This design consideration is thoroughly analyzed in the following sections and optimization strategies are derived for 
both centralized and distributed solutions. Section II includes related work and provides background information on eventbased communication. Section III establishes the networking setup, derives the problem formulation and elaborates on the optimal and heuristic solutions for both the centralized and distributed variations. Section IV provides extensive experimental results that demonstrate the impact of the proposed optimization strategies and Section V concludes this paper.

\section{BACKGROUND AND RELATED WORK}

Event triggering is an operating paradigm conceived specifically for resource-constrained systems. For instance, eventtriggered control has been extensively applied to systems that have limited energy and bandwidth availability [8], [9]. Within this setting, a number of studies have also looked at the networking aspects of event-triggered control by considering mainly the communication delays in the exchange of events [10], [11]. However, in all these cases, devices were assumed to be active and continuously listening over the communication channel for event triggers. Evidently, such an approach only allows gains in channel utilization and penalizes energy efficiency.

The envisioned event-based communication framework consists of three main elements:

Behavior Modeling: Measurement data is used to extract the system behavior model, [12], [13]. This model is used by the local host to detect abnormal changes. It is also passed to remote hosts (i.e., other IoT devices) that use it to estimate the system state when no communication takes place.

Event Detection: Events are triggered whenever the actual system behavior is different from that anticipated by the model. A variety of different techniques can be used to detect these changes. Thresholding is among the simplest approaches, while parity checks, state observers, and other residuals are among the more advance alternatives [14]. Importantly, the sensitivity of event detection (and thus the number of triggered events) is governed by the targeted tracking accuracy in monitoring and the stability requirements in controlling the system at hand.

Event Handling: Triggered events can be used to take local actions (e.g., control the system, update system information, etc.) and are also communicated to remote hosts in order to inform of the event occurrence.

To aid understanding, Figure 1 depicts an arbitrary scenario with two systems managed by three devices. As shown in the figure, devices can manage different parts of one or more systems that are interrelated as indicated in [15] and [16]. In either case, events are communicated whenever the system state is different from that prescribed by the nominal model. The local host compares the estimated state of the model to the actual system state and if an abnormal event is detected it gets communicated to all other devices registered to receive updates. At every other time, the model is used to approximate the system state by both the local and remote hosts and no communication is necessary. A more detailed discussion on the proposed approach can be found in [17] whereby a practical example is used to aid understanding.

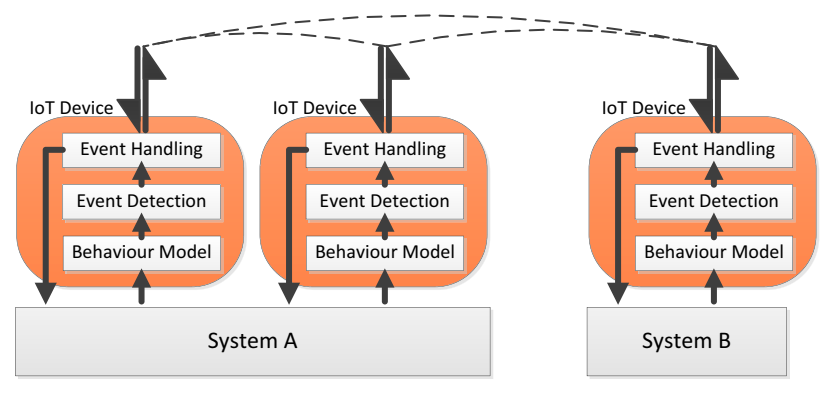

Fig. 1. The network setup under consideration.

In this work, emphasis is placed on the networking aspect of event-based communication, whereby communication circuitry remains asleep unless a triggered event needs to be exchanged. In the latter case, the device powers up and waits until devices registered to receive the events, wake up and establish a connection ${ }^{1}$. The major difference between periodic- and event-based communication is that periodic wake-ups offer a predetermined bound on the time that devices need to wait before a connection is established. In this way, exact performance targets exist and absence of any connection can indicate a faulty operation.

With event-based communication on the other hand, the waiting time is only probabilistically bounded. This is due to the fact that a wake-up may never occur if the system behavior closely follows that described by the model at hand. Nevertheless, increasing the detection sensitivity (and thus the number of triggered events) can limit the waiting time and also reduce the energy consumption for all those other devices that want to established a connection and exchange event messages. However, a device's energy consumption increases proportionally to the number of triggering events, and thus this tradeoff should be properly addressed in order to maximize the network-wide energy efficiency. The following section examines in detail this tradeoff and both optimal and heuristic solutions are provided.

\section{EVEnT-BASED NETWORKING}

A network $\mathcal{N}=\{1, \ldots, N\}$ of devices is considered. Let $\mathcal{Z}(i), i \in \mathcal{N}$ be the subset of nodes in the network that are registered to receive event triggers from device $i$ and let $S(i)$ be the minimum number of triggering events necessary to achieve the desired tracking accuracy and/or to guarantee control stability. Increasing the volume of triggering events above $S(i)$ would result to shorter inter-event times (and thus improved accuracy) at the expense, however, of increased device energy consumption.

Similar to the operation described by upcoming IoT standards (some of which are listed in Section I), whenever an event is triggered by node $i$, its communication circuitry is powered on, a wake-up broadcast beacons its availability, and a connection is established by any of the available nodes in set $\mathcal{Z}(i), i \in \mathcal{N}$ in order to exchange event messages.

\footnotetext{
1 As detailed in the standards discussed in Section I, a wake-up broadcast signals connection initiation.
} 
Node $i$ listens over the wireless channel until all registered devices become available for connection establishment and successfully receive event messages. Thereafter, node $i$ powers down its communication circuitry to conserve energy and the process repeats whenever a new event is detected.

Let $A$ be the duration of time over which recurrent patterns occur for the system to be managed and let $\tau$ be the event transmission time. As an indication, current IoT-enabled communication protocols (such as IEEE802.11ah and LTEMTC mentioned in the Introduction) recommend duty cycles of $\frac{\tau}{A}$ in the range of $1 \%$ or less to improve battery lifetime of embedded devices [18]. Moreover, the power consumption $P_{T x}$ is assumed to be the same for both transmit/receive operations and for listening over the wireless channel while waiting for an event interrupt to be exchanged (as shown in [19] for the communication circuitry of mobile devices).

Let variable $s_{i}, i \in \mathcal{N}$ indicate the number of events to be optimized in order to achieve the maximum network-wide energy efficiency. Then, assuming a uniform distribution of event triggers in the interval $A$, the mean waiting time for all nodes in $Z(i)$ is $\frac{A}{2 \times s_{i}}$, while the total energy consumption for the exchange of $s_{i}$ events between nodes $\{i, j\} \in \mathcal{N}$ can be expressed as follows:

$$
e(i, j)=s_{i}\left(\frac{A}{2 \times s_{j}}+\tau\right) P_{T x}, \forall j \in \mathcal{Z}(i)
$$

As described by eq. (1), to minimize the waiting time, all remote hosts registered to receive events from device $i$ (i.e., nodes in set $\mathcal{Z}(i)$ ) need to increase their number of triggered events. This, however, will increase their own energy consumption and thus $s_{i}, \forall i \in \mathcal{N}$ should be optimized to achieve network-wide energy efficiency gains.

\section{A. Centralized, Optimal Formulation}

A mathematical programming formulation is derived in problem $(P 1)$ that computes the optimal number of event triggers that each device should set in order to minimize the overall energy consumption.

$$
\begin{aligned}
& \text { (P1) } \min \sum_{i=1}^{N} \sum_{j \in \mathcal{Z}(i)} e(i, j) \\
& \text { s.t. } s_{i} \geq S(i), \quad s_{i} \in \mathbb{Z}, \quad \forall i \in \mathcal{N}
\end{aligned}
$$

Problem $(P 1)$ is a fractional integer program with objective function (2) minimizing the overall energy consumption, while constraint (3) ensures that the minimum number of triggered events is satisfied. It should be noted here that while singleratio fractional problems can be easily linearized with the Charnes-Cooper transformation, $(P 1)$ expresses a sum-ofratios that classify it as a hard optimization problem. Also, since complete information of all $S(i), i \in \mathcal{N}$ values is needed in $(P 1)$, it can only be solved in a centralized manner.

\section{B. Distributed Relaxation}

A distributed approach to solving $(P 1)$ is considered herein. To ease readability, let $B=P_{T x} \frac{A}{2}$ and $\Gamma=P_{T x} \tau$. Then $(P 1)$ can be rewritten as follows:

$$
\begin{aligned}
& (\mathrm{P} 2) \min \sum_{i=1}^{N} \sum_{j \in \mathcal{Z}(i)}\left(B \frac{s_{i}}{s_{j}}+\Gamma s_{i}\right) \\
& \text { s.t. } s_{i} \geq S(i), \quad s_{i} \in \mathbb{Z}, \quad \forall i \in \mathcal{N}
\end{aligned}
$$

Considering variables $s_{i}$ as monomials, the division of two monomials as expressed by the first term of the objective function in (4) is also a monomial and the sum of the two monomials is a posynomial function (i.e., the combination of "positive" and "polynomial"). In this way, $(P 2)$ can be viewed as an integer geometric program (GP) which is still a computationally hard problem to solve. Nevertheless, ignoring the integer constraints in (5) provides a simple heuristic solution. We consider this heuristic to devise a distributed GP solution to $(P 2)$. In this solution, each device computes its own local optimization and the resulting solution is exchanged between coupled nodes (i.e., devices registered to receive event triggers) in order to coordinate their computations.

Let $y_{i}=\ln \left(s_{i}\right)$ and hence $s_{i}=e^{y_{i}}$. With this change in variables, the problem can be expressed as follows:

$$
\begin{aligned}
& \text { (P3) } \min \sum_{i=1}^{N} \sum_{j \in \mathcal{Z}(i)} \frac{B e^{y_{i}}}{e^{y_{j}}}+\Gamma e^{y_{i}} \\
& \text { s.t. } y_{i} \geq \ln (S(i)), y_{i} \in \mathbb{R}, \forall i \in \mathcal{N}
\end{aligned}
$$

Auxiliary variables $y_{i j}$ are then introduced for the coupling of individual solutions (computed locally by each device) and extra equality constraints are used to impose consistency between the variables as shown in $(P 4)$ below.

$$
\begin{aligned}
\text { (P4) } \min \sum_{i=1}^{N} \sum_{j \in \mathcal{Z}(i)}\left(B e^{y_{i}-y_{j}}+\Gamma e^{y_{i}}\right) \\
\text { s.t. } y_{i j}=y_{j}, \forall j \in \mathcal{Z}(i) \\
\quad y_{i} \geq \ln (S(i)), y_{i} \in \mathbb{R}, \forall i \in \mathcal{N}
\end{aligned}
$$

Notably, in $(P 4)$ the coupling of variables has been moved from the objective to the constraints using the local variables $y_{i j}$ and consistency is maintained by the constraints expressed in (9). The Lagrangian formulation of $(P 4)$ can then be expressed as follows:

$$
\begin{aligned}
& (\mathrm{P} 5) \min \sum_{i=1}^{N} \sum_{j \in \mathcal{Z}(i)}\left(B e^{y_{i}-y_{j}}+\Gamma e^{y_{i}}\right) \\
& +\sum_{i=1}^{N} \sum_{j \in \mathcal{Z}(i)} \gamma_{i j}\left(y_{j}-y_{i j}\right) \\
& \text { s.t. } y_{i} \geq \ln (S(i)), y_{i} \in \mathbb{R}, \forall i \in \mathcal{N}
\end{aligned}
$$

where $\gamma_{i j}$ are the Lagrangian multipliers (or consistency prices) and the Lagrangian subproblem for each $i \in \mathcal{N}$ would be $L_{i}: \sum_{j \in \mathcal{Z}(i)}\left(B e^{y_{i}-y_{j}}+\Gamma e^{y_{i}}\right)+\left(\sum_{j \in \mathcal{Z}(i)} \gamma_{j i} y_{i}\right)-$ $\sum_{j \in \mathcal{Z}(i)} \gamma_{i j} y_{i j}$. Therefore, each subproblem can be computed in a distributed way and in parallel by each device. Moreover, the Lagrangian multiplier problem $L^{*}=\max _{\gamma} L(\gamma)$ should also be solved to obtain optimal $\gamma$ values for problem $(P 5)$. Since problem $(P 4)$ is convex, the Lagrangian multiplier 
problem is guaranteed to solve $(P 5)$ to optimality.

The subgradient iterative optimization procedure is the most general method to solve the Lagrangian multiplier problem through the sequential improvement in the $\gamma$ estimates. Let $\gamma^{0}$ be any initial choice of the Lagrangian multiplier vector. Then, the subsequent values $\gamma^{t+1}$ for $t=1,2, \ldots$ can be computed as follows:

$$
\gamma^{t+1}=\gamma^{t}+\delta_{k}\left(y_{j}^{t}-y_{i j}^{t}\right)
$$

where $\delta^{t}$ is the step size at the $t^{\text {th }}$ iteration. Of course, an appropriate step size should be chosen to ensure that eq. (13) solves the Lagrangian multipliers problem. For instance, setting $\gamma^{t}=1 / t$ ensures that the procedure does converge since $\gamma^{t} \rightarrow 0$ and $\sum_{l=1}^{t} \gamma^{t} \rightarrow \infty$ as $t$ increases.

\section{Distributed Heuristic}

The Lagrangian multiplier problem described in the previous section only solves the integer relaxation of problem $(P 1)$. Hence, the optimal value of the relaxed GP is a lower bound on the optimal value and simply rounding each of the $s_{i}, i \in \mathcal{N}$ variables to the nearest integer, in general, works poorly. In addition, the subgradient iterative procedure that solves this problem requires that at each step every device: $i$ ) synchronously exchanges its Lagrangian multipliers, $i i)$ computes the Lagrangian subproblem using only local variables $y_{i}$ and $y_{i j}$, and $\left.i i i\right)$ computes the multipliers for the next iteration, i.e., $\gamma_{i j}^{t+1}$ as defined by eq. (13); before exchanging these multipliers again to initiate the next iteration. It is therefore clear that this approach requires extensive communication and its iterative synchronous nature is in contrast with the overall event-based communication paradigm. Nevertheless, the approach provides good insights on how the $s_{i}$ variables should be optimized. In accordance, a heuristic solution is developed to optimize the $s_{i}$ values while maintaining integer solutions.

Similar to the subgradient procedure described previously, the idea is to create an update rule for the $s_{i}$ values based on the condition of reducing the normalized energy consumption between a particular device $i$ and the corresponding nodes in set $Z(i)$. To do so, let $\Delta_{o}(\theta)$ be the energy consumption difference of increasing $s_{i}$ by $\theta$ units, expressed as follows:

$$
\Delta_{o}(\theta)=\frac{\sum_{j \in \mathcal{Z}(i)}\left(B \frac{s_{i}+\theta}{s_{j}}+\Gamma\left(s_{i}+\theta\right)\right)-\sum_{j \in \mathcal{Z}(i)}\left(B \frac{s_{i}}{s_{j}}+\Gamma s_{i}\right)}{|\mathcal{Z}(i)|}
$$

Also, let $\Delta_{\nu}(\theta)$ be the energy consumption difference of all nodes $j \in \mathcal{N}$ for which $i \in \mathcal{Z}(j)$ (i.e., the indegree nodes to $i)$ which can be mathematically expressed as follows:

$$
\Delta_{\nu}(\theta)=\frac{\sum_{\substack{j: i \mathcal{Z}(j) \\ j \in \mathcal{N}}}\left(B \frac{s_{j}}{s_{i}}+\Gamma s_{j}\right)-\sum_{\substack{j: i \in \mathcal{Z}(j) \\ j \in \mathcal{N}}}\left(B \frac{s_{j}}{s_{i}+\theta}+\Gamma s_{j}\right)}{\sum_{\substack{j: i \in \mathcal{Z}(j) \\ j \in \mathcal{N}}} 1_{\mathcal{Z}(j)}}
$$

where $1_{\mathcal{Z}(i)}$ is the indicator function defined as 1 if $i \in \mathcal{Z}(j)$ and 0 otherwise.
Then, a simple update rule can be realized as follows:

$s_{i}\left[t_{k}\right]= \begin{cases}s_{i}\left[t_{k-1}\right]+\theta & \Delta_{\nu}(\theta)>\Delta_{o}(\theta) \\ \max \left(S(i), s_{i}\left[t_{k-1}\right]-1\right) & \text { otherwise }\end{cases}$

Unlike the Lagrangian multiplier problem, the updating of the $s_{i}$ variables can be done in discontinuous discrete steps $t_{k}$ using the most recent values that have been received by other devices during event exchange. Moreover, the magnitude of $\theta$ governs how fast the heuristic converges, while the retreat towards the $S(i)$ value when $\Delta_{\nu}(\theta) \leq \Delta_{o}(\theta)$ ensures convergence.

In summary, whenever an event triggers a wake-up of the $i^{\text {th }}$ node, the device takes the following steps: $i$ ) computes $\Delta_{\nu}(\theta)$ and $\Delta_{o}(\theta)$ using a predetermined $\theta$ value, $\left.i i\right)$ executes the update rule to determine the new $s_{i}$ number, and $\left.i i i\right)$ communicates that number within the wake-up broadcast that signals the connection request (which is in line with how IoT communication standards are developed as discussed in Section II). Other nodes that receive that broadcast update their registers with the new $s_{i}$ value. The process repeats whenever a new triggering event occurs in a purely distributed and asynchronous manner. It should be emphasized here that the overhead of the proposed heuristic is merely the addition of the $s_{i}$ value in the broadcast beacon sent whenever the device wakes up (as part of the connection establishment), and its range (and thus bit size) is solely governed by the application scenario.

\section{Simulation Results}

In this section, a performance analysis of both centralized and distributed solutions is provided. Towards that direction, a network of $N=30$ nodes is considered, while the size and elements of set $\mathcal{Z}(i), i \in \mathcal{N}$ is randomly and uniformly selected in the interval $U \sim(0,|\mathcal{Z}(i)|)$. The minimum number of triggering events $S(i), i \in \mathcal{N}$ is also assumed to be uniformly distributed in the interval $U \sim(0,10)$, while the particular time at which these events are triggered is uniformly distributed within $U \sim(0, A)$, where $A=60$ $\mathrm{sec}$ is the window of time that recurrent patterns emerge (as detailed in Section III). A fixed power consumption of $P_{T x}=0.1 \mathrm{~W}$ is assumed for transmit/receive and listening by the communication circuitry.

This network setup has been implemented in Matlab, and so were the formulations of problem $(P 1)$ and the distributed heuristic described in Section III-C (annotated as DH hereafter). Yalmip's BnB (branch and bound) solver has been used to compute $(P 1)$ to optimality. For the results presented below 1000 Monte Carlo simulations have been conducted and average values are used to investigate the performance of (P1) and DH based on the nominal approach whereby no optimization takes place and all nodes trigger their $S(i)$ events only.

Figure 2 depicts the results obtained for solving $(P 1)$ to optimality under varying cardinalities of set $\mathcal{Z}(i)$. The results presented in Figs. 2a and $2 \mathrm{~b}$ show the total number of event triggers above $S(i), \forall i \in \mathcal{N}$ and the total number of nodes that are affected by this increase. As shown in Fig. 2a, a significant number of triggering events are necessary when $\tau$ 


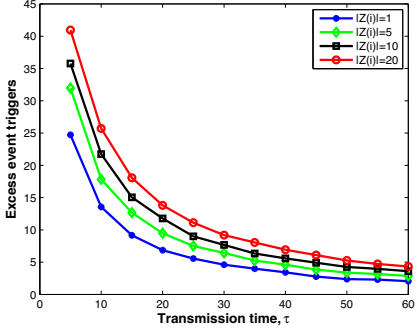

(a)

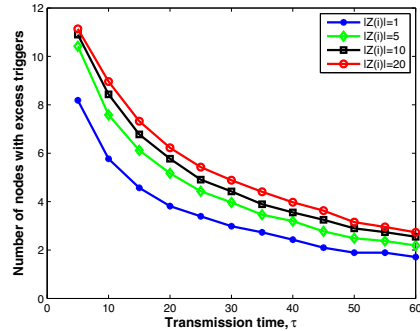

(b)
Fig. 2. Performance analysis of problem (P1) showing (a) the accumulated number of excess event triggers, and (b) the total number of nodes with excess event triggers.

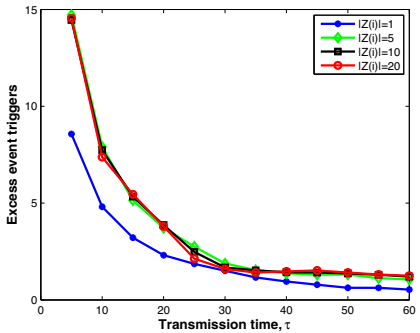

(a)

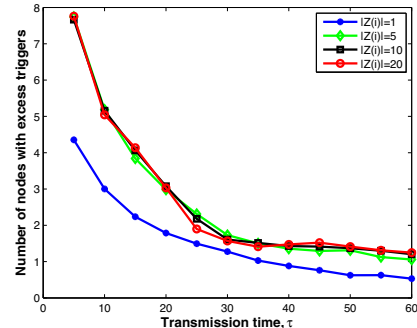

(b)
Fig. 3. Performance analysis of DH showing (a) the accumulated number of excess event triggers, and (b) the total number of nodes with excess event triggers (with $\theta=1$ ).

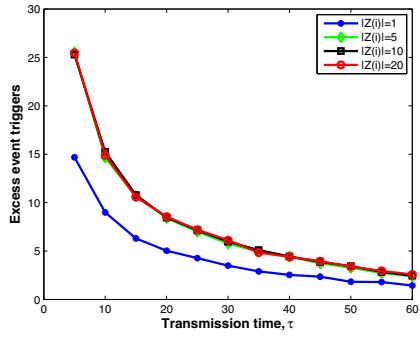

(a)

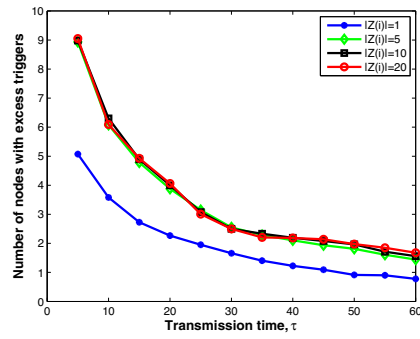

(b)
Fig. 4. Performance analysis of DH showing (a) the accumulated number of excess event triggers, and (b) the total number of nodes with excess event triggers (with $\max \theta$ ).

has a short duration in order to minimize the overall network consumption. Specifically, an increase of approximately $25 \%$ is observed as connectivity increases to $|\mathcal{Z}(i)|=20$. However, this increase only affects a small number of devices (as shown in Fig. $2 \mathrm{~b}$ approximately a third of the participating devices) and not all nodes across the network. Moreover, the number of excess triggers and the number of affected nodes drops exponentially fast when $\tau$ increases, since devices remain awake for longer periods of time and thus waiting is dramatically reduced.

Furthermore, a performance comparison between $(P 1)$ and DH (as described in Section III-C) is performed using two distinct update rules. The first considers merely $\theta=1$ in conditioning the update as described in eq. (16), while the second one considers a more aggressive increment whereby $\theta$ is set to be the maximum number for which condition $\Delta_{\nu}(\theta)>\Delta_{o}(\theta)$ remains true, i.e., $\max \theta$, s.t. $\Delta_{\nu}(\theta)>\Delta_{o}(\theta), \theta \in \mathbb{Z}$.

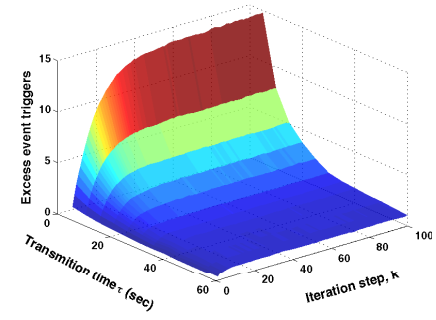

(a)

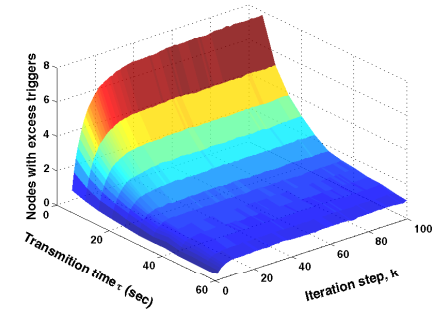

(b)
Fig. 5. Convergence of (a) the accumulated number of excess event triggers, and (b) the total number of nodes with excess event triggers (for the DH with $\theta=1)$.

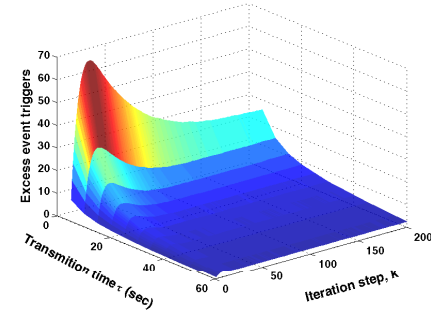

(a)

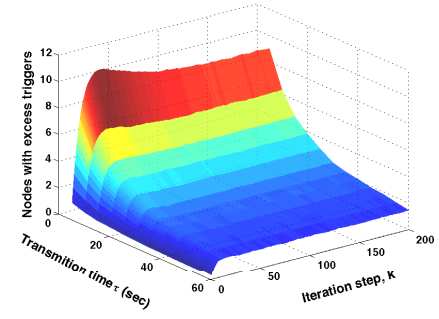

(b)
Fig. 6. Convergence of (a) the accumulated number of excess event triggers, and (b) the total number of nodes with excess event triggers (for the DH with $\max \theta$ ).

Figure 3 depicts the results obtained for $\theta=1$ and Fig. 4 the results for $\max \theta$. As is obvious from the plots, the drop in event triggers, as well as the number of nodes with excess triggers, follow similar trends for DH as in the case with the optimal solution achieved by $(P 1)$. The immediate difference in both cases is that except for the case of $|\mathcal{Z}(i)|=1$, the proposed heuristic achieves similar performance for any arbitrary cardinality of set $\mathcal{Z}(i), i \in \mathcal{N}$. This behavior is attributed to the limited information that the DH has on the degree of connectivity of other nodes in the network and the minimum number of triggered events $S(i)$.

Moreover, Figures 5 and 6 show the convergence evolution of the distributed heuristic for $\theta=1$ and $\max \theta$, respectively. For these results a cardinality of $|\mathcal{Z}(i)|=10$ is considered; however, similar results have been obtained for all other values depicted in the previously documented results. It is evident that for both cases the DH converges to a value that is lower than the optimal (obtained by the solution to $(P 1)$ ). Evidently, this is due to lack of information on the node interactions beyond those established by direct connections. Clearly, such an offset could be corrected by passing information on the $s_{i}$ values beyond the direct reach of each device and incorporating that information into the updating rule expressed in eq. (16). On the other hand, that would increase the signaling overhead and would further impact energy efficiency, since waiting times would inevitably need to increase.

It is also evident that with incrementally small updates achieved with $\theta=1$ convergence is monotonically achieved (exponentially fast), while the aggressive updating achieved with $\max \theta$ overshoots the number of triggered events before establishing convergence. Nevertheless, in both cases only very 


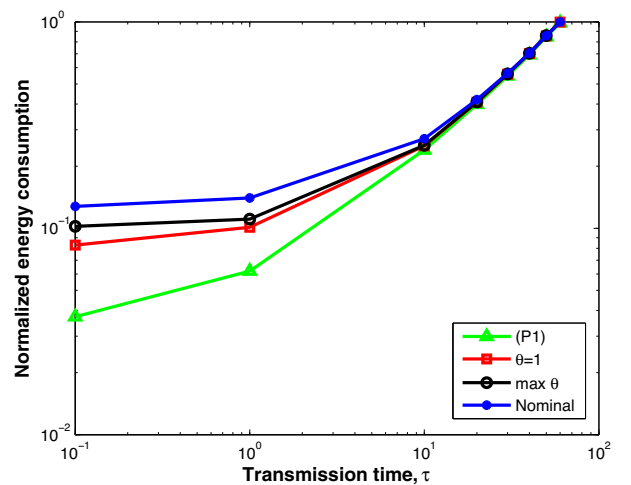

Fig. 7. Energy consumption comparison for the different approaches.

few steps are required for convergence. The clear difference is that the number of event triggers and the nodes affected are considerably higher for $\max \theta$ compared to $\theta=1$, especially when the transmission times are smaller.

Finally, Figure 7 compares the overall energy consumption of all nodes in the network for the event triggering solutions obtained by (P1), the DH approach with $\theta=1$ and $\max \theta$, and the nominal solution where no optimization is carried out (i.e, the energy consumption when the $S(i)$ values are used). As before, the results depicted in Fig. 7 consider a cardinality of $|\mathcal{Z}(i)|=10$, however, similar trends are observed for all other values assumed in Figs. 2, 3 and 4. As shown in Fig. 7, the optimal solution provides significant improvements in energy consumption compared to the nominal solution, especially when transmission times are smaller. For instance, when the transmit time durations are in the order of a few hundred milliseconds to seconds, a gain of more than $50 \%$ is observed compared to the nominal solution. Clearly, higher gains would be obtained for shorter transmission times.

As expected, the improvements of $\mathrm{DH}$ with $\theta=1$ and $\max \theta$ are significantly lower than those obtained by the optimal solution. More interesting is the fact that with $\theta=1$ the energy efficiency gains are marginally better than those achieved by $\max \theta$. Hence, even though $\max \theta$ converges to a solution with higher triggering events (which closely follows the solution achieved by (P1) as shown in Fig. 2 and 4), these triggering events increase the overall network consumption instead of decreasing it. As such, $\theta=1$ suffices as the best alternative DH approach to achieve energy efficiency gains in the distributed setup, while (P1) achieves the maximum energy efficiency gains in the centralized regime where optimal decisions can be effectively made.

\section{Conclusions}

Event triggering is emerging as an enabling paradigm to achieve efficient operation for IoT devices. This work has investigated the networking aspects of event-based communication and more specifically it has looked into the tradeoff between the number of triggered events and the waiting that needs to take place before a connection can be established. Mathematical programming formulations have been derived for both centralized and distributed solutions. An efficient heuristic has also been proposed to achieve a distributed solution that adheres to the upcoming IoT communication standards. Extensive simulation results demonstrate that such an optimization is necessary to minimize the overall network energy consumption (with up to $25 \%$ increase in the number of event triggers when solving (P1) to optimality compared to the nominal approach) while the proposed heuristic has shown to have good performance with exponentially fast convergence.

Future work aims to investigate in more detail the topology characteristics for those nodes that observe an increase in their minimum number of event triggers. Real-world testbeds are also in the works to test the proposed networking approach.

\section{ACKNOWLEDGMENTS}

This work is supported by the European Research Council under the Advanced Grant FAULT-ADAPTIVE ERC-2011AdG-291508.

\section{REFERENCES}

[1] J. Rifkin, The Zero Marginal Cost Society: The Internet of Things, the Collaborative Commons, and the Eclipse of Capitalism, Palgrave Macmillan, Apr. 2014.

[2] Ericsson, Mobility Report: On the Pulse of the Networked Society, Ericsson, June 2015.

[3] K. Christensen, et al., "IEEE 802.3az: The Road to Energy Efficient Ethernet", IEEE Communications Magazine, 48(11):50-56, Nov. 2010.

[4] E. Khorov, "Low Power Wi-Fi: How IEEE 802.11ah is Transforming M2M (Tutorial)", IEEE ISWCS, Aug. 2014.

[5] Qualcomm, "LTE MTC: Optimizing LTE Advanced for Machine-Type Communications", www.qualcomm.com/lte-mtc, Nov 2014.

[6] C. Cassandras, and S. Lafortune, Introduction to Discrete Event Systems, Springer, 2nd Ed., 2008.

[7] H. Hu, Y. Wen, T.S. Chua, and X. LI, "Toward Scalable Systems for Big Data Analytics: A Technology Tutorial”, IEEE Access, 2:652-687, June 2014.

[8] W. Heemels, K.H. Johansson, and P. Tabuada, "An Introduction to Eventtriggered and Self-triggered Control", IEEE Conference on Decision and Control, Dec. 2012.

[9] M. Lemmon, "Event-Triggered Feedback in Control, Estimation, and Optimization", Networked Control Systems - Lecture Notes in Control and Information Sciences, 406:293-358, 2010.

[10] M. Zhong, and C. Cassandras, "Asynchronous Distributed Optimization With Event-Driven Communication", IEEE Transactions on Automatic Control, 55(12):2735-2750, Dec. 2010.

[11] W. Heemels, A.R. Teel, N. Wouw, and D. Nešić, "Networked Control Systems with Communication Constraints: Tradeoffs between Transmission Intervals, Delays and Performance", IEEE Transactions on Automatic Control, 55(8):1781-1796, Aug. 2010.

[12] M. Blanke, M. Kinnaert, J. Lunze and M. Staroswiecki, Diagnosis and Fault Tolerant Control, Springer, Ed. 2, 2006.

[13] K. Slavakis, G. Giannakis, and G. Mateos, "Modeling and Optimization for Big Data Analytics: (Statistical) Learning Tools for Our Era of Data Deluge ", IEEE Signal Processing Magazine, 31(5):18-31, Sept. 2014.

[14] R. Isermann, Fault-Diagnosis Systems: An Introduction from Fault Detection to Fault Tolerance, Springer, 2006.

[15] J.A. Stankovic, "Research Directions for the Internet of Things", IEEE Internet of Things Journal, 1(1):3-9, Feb. 2014.

[16] C. Perera, A. Zaslavsky, P. Christen, and D. Georgakopoulos, "Context Aware Computing for The Internet of Things: A Survey”, IEEE Communications Surveys \& Tutorials, 16(1):414-454, 2014.

[17] P. Kolios, C. Panayiotou, and G. Ellinas, "Tracking Trip Changes with Event Triggering", IEEE Intelligent Transportation Systems Conference. Sept 2015.

[18] M. Dohler, D. Boswarthick, and J.A. Zarate, "Machine-to-Machine in Smart Grids \& Smart Cities: Technologies, Standards, and Applications", IEEE Globecom, Dec. 2012.

[19] K. Pentikousis, "In Search of Energy-Efficient Mobile Networking", IEEE Communications Magazine, 48(1):95-103, Jan. 2010. 